\title{
Telemonitoring for Patients with Heart Failure
}

Introduction: Poor treatment adherence is considered the leading cause of readmission and death in patients with heart failure (HF). Telemonitoring aims to provide clinical support and guidance to prevent clinical worsening.

Methods: This is an updated version of a systematic review conducted by Iglis et al. (2015). Randomized clinical trials evaluating the effect of telemonitoring versus usual care on mortality, all-cause and HF-related admissions and quality of life outcomes in patients with HF were selected. PubMed database was searched, and two independent reviewers selected and extracted data. Risk of bias was assessed by the Cochrane Risk of Bias Tool. Statistical analysis was performed in R software. Quality of life data were reported descriptively.

Results: Thirty-four articles comprising 12266 patients were included. Mean ejection fraction was $40 \%$, and patients used angiotensin-converting enzyme inhibitor (ACEI) or angiotensin receptor blocker (ARB). Clinical support ranged from a phone call to videomonitoring, and follow-up time from 6 to 12 months. The most problematic items in risk of bias were allocation concealment and blinding. There was a $18 \%$ reduction (RR: 0.82 [0.73; 0.93], $\left.\mathrm{I}^{2}: 0 \%\right)$ in the risk of death using telemonitoring compared to usual care. HF-related admissions were reduced by $23 \%$ (RR: 0.77 [0.68; 0.88], I': 2\%) with the intervention, but no change was observed in all-cause admissions. Quality of life improved in telemonitored patients compared to usual care patients after 3 and 6 months of follow-up.

Conclusion: Telemonitoring reduces mortality and HF-related admissions, in addition to improving the quality of life of patients with HF.

Keywords: Heart failure; Telemonitoring; Systematic review. 